\title{
Développement des territoires de projet. Quels enjeux pour les politiques rurales?
}

Territorial development projects: what issues for rural policies?

\section{Marielle Berriet-Solliec et Aurélie Trouvé}

\section{(2) OpenEdition}

\section{Édition électronique}

URL : http://journals.openedition.org/economierurale/3959

DOI : 10.4000/economierurale.3959

ISSN : 2105-2581

\section{Éditeur}

Société Française d'Économie Rurale (SFER)

\section{Édition imprimée}

Date de publication : 15 mai 2013

Pagination : 7-19

ISSN : 0013-0559

\section{Référence électronique}

Marielle Berriet-Solliec et Aurélie Trouvé, « Développement des territoires de projet. Quels enjeux pour les politiques rurales ? », Économie rurale [En ligne], 335 | mai-juin 2013, mis en ligne le 15 mai 2015, consulté le 19 avril 2019. URL : http://journals.openedition.org/economierurale/3959; DOI : 10.4000/ economierurale.3959 


\title{
Développement des territoires de projet Quels enjeux pour les politiques rurales?
}

\author{
Marielle BERRIET-SOLLIEC, Aurélie TROUVÉ • Agrosup Dijon/CESAER UMR 1041 \\ berriet@dijon.inra.fr ; atrouve@dijon.inra.fr
}

Cet article part du constat du renforcement des formes de territorialisation dans le domaine du développement rural et en particulier des " territoires de projet ", dont le programme européen Leader est emblématique. Il en propose une grille de lecture des objectifs et modalités d'intervention, reposant sur les deux critères d'efficacité économique et d'équité. Il croise une littérature théorique et empirique sur la territorialisation des politiques et des travaux de recherche en cours sur l'analyse des politiques de développement rural. In fine, ces territoires de projet apparaissent comme des vecteurs de renouvellement de l'efficacité économique, mais également de remise en cause de la fonction redistributrice de l'État à destination des espaces ruraux.

MOTS-CLÉS : territoire, projet, développement rural, Leader, politique publique

\section{Territorial development projects: what issues for rural policies?}

This article highlights the territorialization of policies and presents a framework to analyse the rural development projects, Leader being a key program. An analytical framework of objectives and implementation is proposed, depending on two criteria: economical efficiency and equity. Theoretical and empirical litterature on policies territorialization, then research studies on rural development policies, are used. Finally, these rural development projects seem to favour a renewal of economic efficiency, but weakens the redistribution function of the State. (JEL: Q18, H50, R58).

KEYWORDS: territory, project, rural development, Leader, public policy

C ette territorialisation est marquée par le renforcement des politiques dites « de territoires de projet ». Dans le domaine du développement rural, Leader (Liaison entre les actions du développement des économies rurales) en constitue un programme phare au niveau européen. Depuis les années 1980 , ces politiques permettent d'afficher une modernisation des interventions publiques ; toutefois, leur développement rencontre des réticences de la part de certains acteurs institutionnels. Ainsi, beaucoup de pays, au premier rang desquels la France et les nouveaux États membres, ont demandé, dans la négociation de la nouvelle programmation du second pilier de la PAC consacré au développement rural, la suppression de la réserve budgétaire permettant de financer les territoires considérés comme «bons élèves » de Leader. Les craintes de ces acteurs recoupent parfois des analyses critiques de la littérature, notamment en termes d'inégalités sociales.

Ce papier revient tout d'abord sur l'émergence des territoires de projet dans les politiques de développement rural, puis en propose une grille d'analyse qui repose sur les deux critères d'efficacité économique et d'équité. Il fait l'hypothèse que ces territoires de projet introduisent de nouvelles formes d'efficacité économique mais tendent à exacerber les inégalités sociales et à s'opposer à l'objectif d'équité. Ces deux hypothèses s'appuient sur la littérature théorique et empirique, sur des travaux de recherche en cours sur des politiques 
régionales de développement rural (BerrietSolliec, Chabé-Ferret, 2011) $)^{1}$, ainsi que sur des enquêtes réalisées sur des politiques de développement agricole et rural dans des collectivités régionales en France et dans d'autres pays européens (Trouvé, 2007). L'ensemble des régions étudiées sont la Bourgogne, l'Auvergne, l'Émilie-Romagne, les Pays-de-la-Loire, Rhône-Alpes, l'Alsace, le Schleswig-Holstein, la Bavière, la Hesse, l'Écosse et Nord-Angleterre. Cet article a extrait de ces travaux les résultats des enquêtes empiriques portant spécifiquement sur les territoires de projet, afin de comprendre en quoi ces territoires sont plus ou moins guidés par la recherche d'efficacité ou d'équité.

\section{Le renforcement des territoires de projet dans les politiques de développement rural}

\section{Pourquoi une intervention publique en faveur des espaces ruraux ?}

Du point de vue économique, l'intervention publique en faveur des espaces ruraux repose sur deux arguments principaux : (i) l'équité ou logique redistributrice, afin de répondre à des inégalités sociales issues de la localisation rurale ; (ii) l'efficacité économique ou logique allocative, la présence d'activités en milieu rural pouvant contribuer à améliorer l'intérêt général défini par un optimum social (Rawls, 1997 ; Sen, 2000 ; Aubert, Gaigné, 2003). Dans le premier cas, il peut être fait référence à un critère de justice sociale, l'État devant

1. Cet article s'appuie sur les travaux issus d'un programme de recherche interrégional (Regiab, 2009-2011), coordonné par M. Berriet-Solliec et $\mathrm{S}$. Chabé-Ferret, co-financé par les régions Bourgogne et Auvergne et l'Institut national de recherche agronomique dans le cadre des programmes partenariaux Pour et sur le développement régional (PSDR). compenser les handicaps des espaces ruraux s'opposant à l'égalité des chances dans l'accès aux biens et services primaires ou entraînant des surcoûts de production liés à l'éloignement (maintien d'une tarification uniforme des services publics, d'équipements publics collectifs en milieu rural...). Dans le second cas, l'accent est mis sur la compensation des défaillances de marché pour atteindre une allocation optimale des ressources, par exemple en prenant en compte les effets externes négatifs liés à l'agglomération (coûts de pollution et de congestion dans la localisation d'arrivée, coûts de désertification, d'approvisionnement pour les agents restants dans la localisation d'origine). Les autorités centrales peuvent donc être amenées, du point de vue de l'efficacité économique, à favoriser la dispersion des activités, à partir d'un certain niveau d'agglomération.

Les travaux en économie géographique ont ainsi contribué à introduire cette dimension spatiale au travers de l'analyse des processus de concentration et de dispersion des activités et des populations (Krugman, 1991). Tant à l'échelle nationale qu'européenne, ces critères d'équité et d'efficacité économique ont conduit à la définition de zonages. Par exemple, la dotation de solidarité rurale relève de cette logique. Cependant, la recherche d'un compromis entre objectifs sociaux et économiques est peu analysée, alors que l'analyse de cette recherche de compromis est au cœur des approches institutionnalistes (Chavance, 2007). Nous proposons donc, en nous appuyant d'une part sur une grille de lecture croisant équité et efficacité et d'autre part sur une approche institutionnaliste, d'analyser les processus de territorialisation des politiques.

\section{Qu'entendre par « territoire de projet " ?}

La littérature sur les territoires de projet s'est développée, depuis les années 
RECHERCHE

1990 jusqu'à aujourd'hui, en partie sous l'impulsion des économistes dits « de la proximité » (Colletis, Pecqueur, 1993 ; Colletis et al., 1999 ; Angeon, Bertrand, 2009). Les géographes et sociologues des réseaux se sont également intéressés à ces thématiques, par exemple en essayant de comprendre comment se constituent des systèmes d'acteurs et sur la base de quels types de liens émerge un projet collectif territorialisé (Maurel et al., 2010). C'est principalement au premier groupe de travaux en économie que nous nous référons ici. Nous entendons par «territoire de projet », une portion d'espace dont la géométrie peut varier selon le projet dont il est porteur (Angeon, Bertrand, 2009). Selon ces auteures, la délimitation du territoire de projet repose sur une combinaison variable de logiques géographiques (logique de voisinage) et organisationnelles (logique d'appartenance aux valeurs communes d'une structure, logique d'adhésion à un projet).

Le territoire de projet est donc un territoire-construit à géométrie endogène (Berdot, Léonard, 2006), qui varie selon le problème productif et le projet en question (Pecqueur, 2001). Il se distingue du territoire-donné qui correspond aux frontières politico-administratives stables. Ce territoire-construit correspond à un lieu institué ou en cours d'institutionnalisation et en perpétuelle redéfinition, correspondant à un processus d'auto-organisation (Gaignette, Nieddu, 2000) : c'est le «territoire identifié comme espace de gestion, dont la délimitation s'établit par type de problème à traiter », se substituant à la « représentation de pouvoirs politiques ordonnés suivant un principe excluant tout chevauchement ou superposition » (Duran, Thoenig, 1996).

Au delà de ces définitions théoriques, l'analyse des origines des processus de territorialisation permet de mettre en évidence, non pas une opposition frontale entre ces deux types de territoires mais un lien plus ou moins étroit selon les configurations spatiales ou sociologiques. Dans le cas français, Rivet (2007) distingue ainsi deux origines au mouvement de territorialisation de l'action publique. En premier lieu, la territorialisation caractérise les tentatives, dès les années 1960, de restaurer la crédibilité du politique, en renforçant des échelons politico-administratifs locaux (création de la Datar en 1963, mise en place de la politique dite de « rénovation rurale », définition des commissariats de massifs...). Ce premier processus se traduit par un mouvement de décentralisation avec transfert de souveraineté à des entités administratives à faible charge identitaire, avec maintien du principe et des règles de la démocratie représentative. Mais la territorialisation désigne, en second lieu, un « appétit de terroirs, de racines, de traditions que manifestent des réseaux intervenant dans des champs très divers » (Rivet, 2007), ce second processus n'étant pas indépendant du premier. Il s'ensuit le développement de territoires dits « de projet » se traduisant par des tentatives de structuration d'acteurs locaux, privés et/ou publics, autour d'une identité territoriale et de projets locaux, tentatives qu'ont commencé à soutenir les pouvoirs publics.

Dans le cas des dispositifs de développement rural que nous étudions et qui résultent de la traduction locale de dispositifs européens et/ou nationaux, le « territoire de projet » est un espace de référence qui a du sens pour l'action des acteurs, en particulier dans la mesure où il correspond à la réponse à un appel à projet lancé par un échelon politico-administratif (Union européenne, État, collectivités). La construction de ce territoire de projet est donc le plus souvent motivée par une incitation financière et l'obtention de crédits publics, parfois par la mise en cohérence de différentes procédures et sources de financements multiples. Elle implique un 
accord négocié sur des objectifs d'action, l'engagement sur un calendrier de moyen terme et des contributions des partenaires engagés en moyens financiers et humains (Angeon, Bertrand, 2009).

\section{Le développement des territoires de projet}

Les collectivités territoriales peuvent être considérées comme un catalyseur de cette territorialisation, en transférant des subventions et des compétences à ces territoires construits, qui décident de l'éventail d'actions à mener sous réserve de validation par les collectivités (Berriet-Solliec, Chabé-Ferret, 2011). C'est par exemple le cas depuis 2004 des Contrats territoriaux uniques en Pays-de-la-Loire qui drainent des sommes importantes (environ $6 \%$ du budget annuel du Conseil régional de 2005 à 2007 (Trouvé, 2007)). De même, Rhône-Alpes met en œuvre des Contrats de développement durable, constitués notamment d'un volet agricole et rural, les Programmes stratégiques agricole et de développement rural (PSADER). Dans de nombreuses autres régions européennes, les territoires de projet constituent une composante importante des politiques de développement rural. C'est le cas des programmes de rénovation des villages et de développement régional des Länder allemands ou des Proder en Catalogne. Néanmoins, les modalités de mise en place de ces territoires de projet (nature des porteurs de projet, procédures éventuelles de sélection et échelons de décision et de gestion) varient sensiblement selon les États membres et les collectivités qui les portent.

L'État français a participé également de ce développement des territoires de projet avec la mise en place de dispositifs aux contours certes très différents (systèmes locaux de production en 1999, pôles de compétitivité et pôles d'excellence rurale en $2005 . .$. ) mais qui correspondent tous à la mise en concurrence d'espaces en vue de financer les projets les plus performants au regard de critères de sélection définis par le financeur.

Il faut cependant souligner l'influence particulière des politiques européennes avec les programmes Leader, qui associent les niveaux national, régional et infrarégional (Shucksmith, 2000 ; Dwyer et al., 2007). Créés en 1991 à titre expérimental, ces programmes sont d'abord dotés d'un faible budget (Berriet-Solliec et al., 2001). Leader s'est ensuite vu renforcé budgétairement et politiquement jusqu'à devenir, depuis 2007, un des quatre axes du second pilier de la Politique agricole commune ${ }^{2}$. Des régions européennes en ont alors fait un axe central de leur programme régional de développement rural $^{3}$ sur 2007-2013 : c'est le cas en particulier de l'Irlande du Nord, qui y consacre près de $30 \%$ des fonds européens, pour la diversification des activités et la qualité de vie en zone rurale. Les régions du Schleswig-Holstein, de Sardaigne, des Pouilles, des Asturies et la communauté de Valence y consacrent 10 à $15 \%$ (Trouvé, 2007). Sur initiative des associations environnementales, certains Länder allemands ont récemment encouragé des «Leader nature » réunissant des acteurs divers pour la gestion de la protection de la nature, et autour de cet objectif, la résolution des conflits d'usage en milieu rural. L'ambition affichée par la Commission en intégrant les programmes Leader dans le second pilier de la PAC était bien de

2. Pour la période 2007-2013, 525 millions d'euros sont consacrés en France au programme Leader soit $5 \%$ de l'ensemble des financements publics consacrés au FEADER (Fonds européen agricole et de développement rural). Source : L'Europe s'engage en France. Avec le FEADER, l'Europe investit dans les zones rurales. Union européenne, ministère de l'Alimentation, de l'Agriculture et de la Pêche, octobre 2007.

3. Les programmes de développement rural sont les déclinaisons, nationales ou régionales, du second pilier de la Politique agricole commune. 
le «Leaderiser », entendons par là d'insuffler davantage de démarches ascendantes et intersectorielles, tout en associant divers partenaires privés au sein d'une politique de développement rural essentiellement caractérisée jusqu'à aujourd'hui par sa dimension agricole.

Ainsi, les territoires de projet se sont progressivement imposés, à la fois au niveau des politiques européennes, nationales et des collectivités territoriales, avec parfois la recherche d'une mise en cohérence (en France, par exemple, le ministère de l'Agriculture a donné comme condition sine qua non de faire correspondre pour la programmation 2007-2013 les territoires Leader aux territoires préexistants dans les politiques nationales de développement rural).

\section{Les territoires de projet Une efficacité économique renouvelée?}

Les territoires de projet résultent de la conjonction d'une intervention publique et d'une relative homogénéité économique voire socio-culturelle d'un espace infrarégional. Cette superposition est censée conférer à l'intervention publique une meilleure efficacité, reposant sur une meilleure utilisation de ressources spécifiques et une prise en compte des demandes des acteurs locaux. Elle est aussi censée cibler les dépenses budgétaires sur les projets les plus efficaces.

\section{L'utilisation de ressources spécifiques du territoire}

Le développement des territoires de projet mise sur une compétitivité des entreprises qui s'appuie sur des avantages spécifiques aux territoires (Colletis et al., 1999). Plus précisément, c'est la « rencontre productive » entre le projet d'entreprises et celui d'un territoire qui pourra donner lieu à un ancrage territorial générateur de développement et de co-production de ressources (Colletis, Pecqueur, 1993). Ceci passe notamment par la mobilisation de ressources immatérielles, la capacité de coordination des acteurs locaux et la mise en œuvre d'apprentissages collectifs (Pecqueur, 2001). C'est précisément sur ce processus que veut miser le dispositif Leader. Pour ce faire, le « principe d'ascendance » prôné par ce programme met l'accent sur le recours à l'animation et à l'investissement immatériel. En outre, les programmes Leader défendent l'idée selon laquelle les ressources cognitives du territoire seraient mieux valorisées localement, les acteurs locaux disposant de davantage d'informations et d'une meilleure maitrise des trajectoires de développement de leurs territoires (Shucksmith, 2000).

Ces territoires d'action jouent également sur la capacité de coordination des différents acteurs du développement rural, en soutenant les mises en réseau et décloisonnements, notamment pour la recherche de cofinancements : publics et privés ou entre différents échelons publics. Ces processus d'incitation apparaissent comme des réussites de Leader, mises en exergue lors des sommets européens successifs dédiés au développement rural (Cork, Salzbourg). Malgré tout, les territoires de projet peinent parfois à développer de telles coordinations. L'intégration des territoires de projet au second pilier de la PAC via le programme Leader en est un exemple. Partant du constat que les projets Leader 2000-2006 touchent peu les agriculteurs, les autorités catalanes tirent ainsi un bilan relativement négatif des expériences PRODER (Trouvé, 2007). Plus encore, les politiques de territoires de projet peuvent amener à un éclatement spatial des formes de coordination, au détriment d'actions plus collectives. En utilisant la notion de « rayon de confiance », entendue comme 
une mesure abstraite de la taille du groupe social avec laquelle un individu est prédisposé à coopérer, Callois (2009) conclut qu'un processus de « laisser-faire » qui donnerait libre cours à l'expression endogène des groupes sociaux conduit à un inéluctable mitage de l'espace et à la constitution de territoires construits selon l'importance relative du rayon de confiance. Sauf s'il existe au préalable une forte prédisposition à l'action collective, un tel processus peut être source d'inefficacité. C'est ce qui amène à privilégier des territoires de projet aux frontières préétablies afin, comme le précise la Région Pays-de-la-Loire dans sa plaquette de présentation du Contrat territorial unique, de ne pas favoriser le démembrement de territoires déjà agréés.

\section{La prise en compte de demandes spécifiques aux territoires}

Le développement des territoires de projet mise également sur la capacité d'un groupe d'acteurs locaux à mieux prendre en compte les demandes sociales et environnementales spécifiques aux territoires, la volonté d'y maintenir un tissu économique et social et d'y préserver les ressources naturelles (Berriet-Solliec et al., 2006). Les territoires qui se mettent en place peuvent dès lors être considérés comme vecteurs d'opportunités nouvelles pour l'expression des acteurs locaux de la société civile, portant des revendications sociales et environnementales peu entendues aux échelons supérieurs (Trouvé, 2007). Ainsi, les Contrats territoriaux uniques en Paysde-la-Loire et les Contrats de développement en Rhône-Alpes s'appuient sur des conseils locaux de développement rassemblant des acteurs de la société civile.

Cependant, selon la nature des projets et les règles qui l'orientent, les territoires peuvent également porter des logiques de compétitivité sur les marchés mondiaux, ne donnant pas la priorité aux demandes sociales et environnementales spécifiques aux territoires, mais à l'insertion économique internationale de ces territoires et aux intérêts des entreprises les plus compétitives sur les marchés, ainsi qu'à la spécialisation des territoires selon leurs avantages comparatifs respectifs (Lovering, 2001). Dans ces conditions, les territoires de projet ne représentent pas une alternative possible aux logiques nationales et européennes, mais au contraire tendent à les reproduire.

Un autre effet pervers résulte du co-financement des politiques. La Région Paysde-la-Loire relève ainsi que « la logique de consommer rapidement les crédits alloués a conduit certains territoires à retenir des opérations de moindre intérêt » (Région Pays-de-la-Loire, 2007). En outre, le principe d'additionnalité des projets Leader (un Euro de Bruxelles appelle un Euro de l'État-membre dont la moitié financée par les collectivités territoriales) a incité les porteurs de projets à s'ajuster sur les priorités régionales. Ainsi, en Poitou-Charentes, plusieurs projets Leader ont proposé des actions en faveur des personnes handicapées, une des lignes politiques fortes du conseil régional. Il en est de même de l'accent mis en Auvergne dans les territoires de projet sur l'accueil des populations, un des axes phares du conseil régional. Ce biais induit par le cofinancement régional apparaît dans certains cas comme un facteur limitant pour le développement d'initiatives innovantes. Par conséquent, certains territoires, avant même d'être soumis au processus de sélection, se sont autocensurés en considérant que leurs actions ne relevaient pas suffisamment des priorités régionales.

\section{Ciblage budgétaire et orientation vers le marché : des gages supplémentaires d'efficacité économique}

S'appuyant sur des analyses économiques qui pointent d'une part le caractère 
RECHERCHE

inefficace des politiques redistributives reposant sur les zonages et d'autre part les excès des politiques d'aides directes (Sapir, 2003), les démarches de projet apparaissent comme un moyen de cibler les efforts budgétaires sur des groupes d'acteurs susceptibles d'utiliser de façon efficace ces fonds publics, tout en générant, par leurs actions, des effets d'entraînement sur le tissu productif local, comme l'estiment Knickel et Jahn (2006) au sujet d'un nouveau programme allemand Regionen Aktiv: "Designing the programme as a competitition encouraged regions to demonstrate greater innovativeness in their development concepts and methods of implementation».

Le développement de ces territoires de projet s'avère alors un moyen de réduire les budgets publics alloués à une politique. La territorialisation est considérée avant tout en termes d'efficience, c'est-à-dire comme moyen de limiter les dépenses financières en concentrant les crédits sur les acteurs considérés comme plus performants dans le sens où ils sont capables, avant le versement de deniers publics, de montrer un projet convaincant pour les gestionnaires de ces aides. Ainsi, confrontée à la réduction de moyens budgétaires pour ses aides agroenvironnementales, la Hesse a décidé de passer de soutiens versés selon une « logique guichet »- pouvant être versés à chaque agriculteur répondant à certaines conditions -, à une « logique projet » permettant de prévoir a priori une enveloppe restreinte. Selon ces modalités renouvelées, il existe une concurrence entre les projets pour bénéficier d'une enveloppe budgétaire limitée. Pour autant, ces affirmations doivent être relativisées. Ainsi, les politiques de territoires de projet peuvent aboutir in fine à des coûts supplémentaires, avec des procédures administratives lourdes pour sélectionner et évaluer les projets. Ces procédures peuvent également prendre le pas sur la dynamique de projet et freiner, voire stériliser des initiatives locales (Fournier, 2000).

L'orientation vers des logiques marchandes est également avancée comme gage supplémentaire d'efficacité économique par les promoteurs des territoires de projet. Cette orientation est facilitée par l'association étroite des acteurs privés à l'élaboration, à la mise en œuvre et au cofinancement des projets. Ce partenariat peut être considéré comme un moyen de réduire le rôle de l'État et de reconfigurer la régulation du secteur dans un «private sector style » (Jones, 1998). De plus en plus d'acteurs privés sont ainsi associés aux décisions et aux financements, privilégiant les pratiques et les objectifs marchands des entreprises privées. Certaines fonctions de l'État sont de fait déléguées à des institutions privées ou mixtes, mieux informées des réalités socio-économiques du secteur privé (Cooke, Morgan, 1998). Cette évolution de l'intervention publique se retrouve dans le rapport Haskins $(2003)^{4}$ : il est explicitement indiqué la nécessité pour l'intervention publique de s'inspirer des pratiques des entreprises privées. La mise en place des programmes Leader en Irlande illustre bien cette évolution. Ainsi, des structures privées (Local Development Companies) ont été mises en place dans ce pays pour venir en appui au montage de projets à l'échelle des territoires, de façon à professionnaliser les groupes locaux et donc à rentabiliser la gestion des fonds Leader.

\section{Une remise en cause de la fonction redistributive de l'État?}

Les territoires de projet peuvent être perçus comme un moyen d'occulter la fonction

4. Commandité par le ministère anglais de l'Environnement, de l'Alimentation et des Affaires rurales pour mettre en place un plan de modernisation de ses politiques. 
redistributrive de l'État, en le cantonnant dans un simple rôle de cocontractant (Kroll, 2002) : ce faisant, l'État n'est plus régulateur mais simple animateur (Cooke, Morgan, 1998) ou facilitateur (Angeon, Bertrand, 2009), il perd la possibilité d'imposer une logique d'équité. Cette remise en cause de la fonction redistributrive de l'État est renforcée par la primauté conférée à l'efficacité économique et par l'orientation vers des pratiques et objectifs marchands, au détriment d'objectifs collectifs (Keating, 1997). Plus précisément, cette partie souligne les risques d'exacerbation des inégalités sociales et la remise en cause de l'équité entre territoires comme entre acteurs au sein de ces territoires.

\section{Remise en cause de l'équité entre territoires... contrecarrée par des stratégies régionales}

Les territoires de projet impliquent généralement la sélection et donc la mise en concurrence des projets entre eux, voire leur conformation à un modèle-type de pratiques (Berriet-Solliec et al., 2006). Inévitablement apparaissent des territoires qui perdent et d'autres qui gagnent, ces gagnants étant ceux qui arrivent à coupler à la fois des dynamiques politique, économique, sociale et culturelle (Angeon, Bertrand, 2009). Par exemple, en Hesse, les projets de rénovation des villages sont sélectionnés à partir des besoins de la communauté mais aussi à partir de l'existence de groupes actifs, capables d'initiatives d'une certaine conception du développement régional. Sont favorisés les villages qui disposent déjà de ressources importantes (matérielles et humaines) et d'une expérience dans l'élaboration de projets. Cette orientation est renforcée par le fait que les mécanismes visant à compenser la mise en concurrence et les inégalités entre espaces locaux sont soit progressivement mis à mal, soit quasi inexistants.
Ainsi, les travaux menés en Auvergne (Callois, 2007, 2009) démontrent, grâce à la mobilisation d'un modèle d'analyse micro-économique, l'importance de ces inégalités dans la dotation en capital social et l'influence des facteurs sociologiques dans la capacité à monter un projet de développement. L'analyse détaillée de quelques exemples de politiques de territoires de projet par des collectivités régionales montre en outre l'avantage qui est donné à des territoires préexistants à un appel à projet donné. C'est le cas du programme Leader. Tel qu'il est appliqué en France sur la période 2007-2013, ce programme favorise explicitement les territoires déjà institués (parcs naturels régionaux, pays déjà structurés - au sens de la Loi Voynet de 1999 sur l'aménagement du territoire). En effet, un tel exercice nécessite des compétences en ingénierie territoriale. Plus largement, l'élaboration d'un projet autour d'une priorité ciblée repose sur des compétences spécifiques (mobilisation de réseaux, organisation de réunions de concertation, pilotage, mise en place d'un dispositif de suivi/évaluation...) dont ne disposent pas tous les territoires.

Pour essayer de contrecarrer cet effet, le second pilier de la PAC prévoit une mesure dite d'appui à l'ingénierie territoriale (mesure 341b). Pour 2007-2013, cette mesure a représenté en moyenne 1,8\% des financements totaux du deuxième pilier (données ministère de l'Agriculture/DGPAAT). Dans beaucoup de régions, ces crédits d'assistance technique ont été orientés vers les territoires qui n'ont pas encore bénéficié de crédits Leader. C'est ainsi que la Bourgogne, pour le montage des candidatures des nouveaux programmes, a proposé un appui financier pour le montage de dossier à hauteur de $30000 €$ par territoire. Dans les faits, cette mesure ne se révèle pas suffisante pour soutenir les territoires marginalisés dans leurs démarches de montage de projet. En effet, l'obstacle 
relève moins de moyens financiers que de présence de personnel qualifié. Pour ce faire, la région Centre a récemment proposé le recrutement de chargés de missions, dans les zones défavorisées du sud de la région.

De ce fait, certaines collectivités territoriales reviennent à des politiques de guichet, par souci de non-exclusion (mais aussi par souci électoral). C'est le cas du Limousin, qui a défendu le fait de retenir tous les projets déposés quelle que soit la qualité de ceux-ci. En Bourgogne, malgré un avis négatif sur certains projets par le groupe des experts régionaux au regard de critères objectifs établis à partir d'une grille de sélection nationale, tous les dossiers ont in fine été retenus à la suite de l'avis du Conseil régional. Les élus ont préféré diminuer l'enveloppe de chacun des projets pour pouvoir faire en sorte que tous en bénéficient. Cet exemple illustre les contradictions d'une politique de projet se rapprochant finalement dans sa traduction concrète, d'une logique de guichet.

Le cas du Conseil régional de RhôneAlpes est également illustratif de la tension qui peut exister entre la volonté de sélectionner des projets et le souci de répartition des fonds publics sur toute une circonscription électorale, en vue d'assoir une légitimité politique. Partant du constat que la région, tout comme les services déconcentrés de l'État à l'échelle régionale, joue à ce jour un rôle relativement restreint de mise en cohérence des mesures de politique agricole et rurale (Berriet-Solliec et al., 2006), le Conseil régional a souhaité mettre en place des Contrats de développement durable en Rhône-Alpes (CDDRA). Ceux-ci apparaissent, au regard de leurs finalités politiques, comme des instruments de légitimité des pouvoirs régionaux, dans une région au périmètre étendu, regroupant huit départements aux espaces hétérogènes (zones de montagne ou de plaine, zones peu urbanisées et grands pôles urbains, agricultures spécialisées ou fortement diversifiées ...). Ces contrats peuvent concerner l'ensemble des territoires rhônealpins, puisqu'ils ne misent pas sur la sélectivité et la mise en concurrence des territoires. Dans ce cas, les politiques de territoires de projet aident la collectivité régionale à s'imposer comme fédératrice d'une entité spatiale et lui confèrent une légitimité démocratique par un transfert partiel des décisions et des financements à des territoires infra-régionaux.

\section{Une remise en cause de l'équité au sein des territoires}

Les territoires de projet interrogent également les inégalités infra-territoriales, ainsi que le fait Shucksmith (2000) à propos des projets Leader et de leur mise en œuvre au Royaume-Uni : ces projets risquent de passer outre l'hétérogénéité sociale des territoires visés et les rapports de force qui s'y expriment. Il souligne le fait que dans les projets dits de «développement endogène », ce sont souvent les élites locales de ces espaces ruraux - les acteurs disposant déjà d'un « capital social » élevé -, qui dominent les processus, ce qui exclut encore davantage les groupes déjà marginalisés. $\mathrm{Au}$ Royaume-Uni, ce sont souvent ces élites qui définissent la culture identitaire locale recherchée dans ces projets ; culture dans laquelle peuvent ne pas se retrouver un certain nombre d'autres acteurs. Ce sont souvent eux qui déterminent les partenaires impliqués, les objectifs et finalement les traits saillants des projets de développement local et rural. Cette domination des élites est renforcée quand les projets se font sur le court terme, ce qui ne laisse pas la possibilité de phénomènes d'apprentissage pour d'autres acteurs. De même, Shucksmith (2000) note au Royaume-Uni que le renforcement des contraintes en termes de cofinancement privé/public et la restriction des budgets publics ont renforcé 
le poids des élites dans les programmes Leader. Ces premiers résultats relatifs à un contexte spécifique britannique se retrouvent dans des contextes français, allemands ou italiens (Berriet-Solliec, ChabéFerret, 2011), mais ils sont à nuancer en fonction de la place des élites locales dans les réseaux locaux et de la mise en œuvre nationale des programmes territoriaux, comme l'illustrent les travaux en Hongrie et en République tchèque des géographes Maurel, Chevalier et Pola (2010).

Il faut ainsi noter que la domination des élites locales dépend des modalités concrètes d'application des projets. Par exemple, certains projets Leader ou d'autres expériences de territoires de projet (comme les «PARC » au Pays-de-Galles) ont fixé non pas des objectifs de résultat en termes de développement économique, mais des objectifs de moyen terme, passant par des processus participatifs et visant à inclure des acteurs généralement exclus (Shucksmith, 2000). Mais même avec de tels objectifs de moyen, la Région Pays-dela-Loire, dans un document d'évaluation de ses Contrats territoriaux uniques, note que les impératifs d'échéances accélèrent le rythme de préparation des contrats et compromettent " une réflexion partagée sur les attentes et besoins réels du territoire » (Région Pays-de-la-Loire, 2007).

Plus généralement, les territoires de projet rejoignent la « cité par projets » de Boltanski et Chiapello (1999), où le projet est considéré comme un rassemblement temporaire de personnes, pour « forger des liens plus durables qui seront ensuite mis en sommeil tout en restant disponibles ». Chaque individu réussit à s'insérer dans des projets s'il est capable de se connecter à des réseaux et pour cela d'avoir des qualités communicationnelles et relationnelles, pour accumuler un fort capital social et d'information. Si ce n'est pas le cas, il risque l'exclusion, qui peut être d'autant plus brutale que les inégalités d'information sont cumulatives. De la même façon, les territoires de projet s'accompagnent inévitablement de risques d'exclusion de tous ceux qui ne réunissent pas les qualités nécessaires pour s'inscrire dans ce type de logique.

\section{Les territoires de projet, vecteurs d'un affaiblissement de l'intervention publique ?}

La territorialisation s'intègre dans un contexte de remise en cause des fondements et des modalités de l'intervention publique dans les champs économique et social (Lovering, 2001). Ce contexte s'accompagne de plusieurs évolutions : (i) un affaiblissement budgétaire et règlementaire de cette intervention ; (ii) une association plus étroite des acteurs privés dans la définition, la mise en œuvre et le financement des politiques, par une mise en concurrence des acteurs et des espaces ; (iii) une primauté de l'objectif de compétitivité par la réduction des coûts ${ }^{5}$. Selon Berdot et Léonard (2006), ce mouvement s'inscrit dans les discours sur le nécessaire changement de fonction de l'État, sur le hollowing out of the state (Angeon, Bertrand, 2009).

Les politiques de développement rural n'échappent pas à ces évolutions. Ainsi, par rapport à la période précédente, l'enveloppe budgétaire européenne allouée au développement rural est réduite sur la période 2007-2013 pour l'ensemble des

5. Ceci au détriment de considérations non marchandes, sociales et environnementales et même si les textes européens mettent en avant la primauté des facteurs de compétitivité liée à l'innovation et les connaissances. 
anciens États membres ${ }^{6}$. En outre, l'ancien objectif 2 de la politique régionale européenne a été converti en objectif de « compétitivité régionale et emploi » et a vu disparaître le zonage prédéfini sur des zones spécifiques en difficulté. Il en résulte une concentration des soutiens des programmes régionaux sur les zones urbaines, jugées les plus compétitives (Aubert, Trouvé, 2008).

Plusieurs éléments soulignés dans cet article montrent ainsi que les mécanismes associés aux territoires de projet correspondent, sous certains aspects, à un affaiblissement progressif de l'intervention publique et à son adaptation aux logiques du marché. En particulier, sur le plan réglementaire, les territoires de projet ne s'accompagnent pas de contraintes sur les acteurs économiques, mais seulement d'incitations à des changements de comportements. Sur le plan budgétaire, le ciblage des dépenses publiques et leur diminution est un argument clé avancé, de même que l'orientation vers les pratiques et objectifs marchands.

Finalement, souvent synonymes de modernisation des modalités de l'intervention publique, les politiques dites de projet soulèvent de nombreuses interrogations relatives aux fondements qui les sous-tendent. Le présent article analyse ces politiques au regard des deux critères d'efficacité économique et d'équité et met en évidence des formes renouvelées d'efficacité économique, reposant sur l'adaptation aux spécificités territoriales. Mais cette hypothèse est nuancée à différents égards. Ainsi, les territoires de projet, associés aux mécanismes de cofinancement et à une plus forte orientation vers la recherche de compétitivité des entreprises, peuvent ne pas

6. En effet, la politique européenne de développement rural se trouve transférée vers le second pilier de la Politique agricole commune, lui-même restreint sur le plan budgétaire et devant répondre aux besoins de l'élargissement. répondre aux demandes spécifiques des territoires. Surtout, ces politiques peuvent exacerber les inégalités entre territoires et entre acteurs au sein de ces territoires, remettant en cause l'objectif d'équité. Plus encore, le développement des territoires de projet, associé à une disparition progressive des zonages de l'intervention publique, pourrait s'accompagner d'un glissement progressif d'un objectif de redistribution territoriale à celui d'efficacité économique, alors même que les territoires disposent de ressources très inégales pour y répondre. Mais là encore, cette hypothèse doit être nuancée par la réalité des politiques mises en œuvre : si les politiques de projets conduisent, dans certains cas, à des processus de sélection, dans d'autres, comme Leader dans certaines régions françaises (Bourgogne, Alsace, Bretagne, Limousin...), il s'avère que les modalités retenues in fine sont peu ou pas sélectives. Le critère de renforcement de la compétitivité par la sélection des territoires les plus performants n'apparaît alors que comme un affichage politique.

Ces premières réflexions appellent des approfondissements et tracent des perspectives. La question posée est aujourd'hui d'analyser le risque d'exacerber les inégalités entre territoires et les modalités de compensation par le renforcement des outils d'accompagnement de ces territoires de projet (formation, appropriation par les acteurs...). Il s'agit également d'approfondir les questions relatives à l'équité, qu'elle soit entre territoires ou entre acteurs de ces territoires : peut-on s'affranchir de la fonction redistributive de l'État et du maintien de mesures de péréquation et de politiques aux échelons supra-territoriaux ?

L'affirmation et l'intégration récentes de Leader dans la politique de développement rural (second pilier de la PAC) ouvrent également des perspectives d'analyse sur les effets de cette intégration et leur comparaison entre différents États membres de 
l'Union européenne. Une analyse critique des territoires de projet pourrait être également poursuivie en introduisant davantage, au-delà des critères d'efficacité économique et d'équité, la question des rapports de pouvoir qui se jouent entre acteurs publics et privés, de même que leurs incidences en termes démocratiques. Ainsi, les territoires de projet peuvent être considérés comme sources de légitimité démocratique (cas des territoires de citoyenneté au
Brésil) et d'une re-politisation par le biais du local, c'est-à-dire d' « un retour au politique, à l'action de proximité par un transfert aux collectivités territoriales » (Lamarche, 2008). Dans un contexte de réforme territoriale et de redéfinition des contours des interventions des institutions locales, les territoires de projet s'avèrent dès lors être un moyen potentiel, pour une collectivité, d'asseoir sa propre légitimité et sa sphère d'influence politique.

\section{REFERENCES BIBLIOGRAPHIQUES}

Angeon V., Bertrand N. (2009). Les dispositifs français de développement rural : quelles proximités mobilisées ? Géographie, Économies et Sociétés, 2009/2, vol. 11, p. 93-114.

Aubert F., Gaigné C. (2003). Les espaces ruraux et la politique d'aménagement du territoire. INRA Sciences Sociales, vol. 1-2, $\mathrm{n}^{\circ} 3$.

Aubert F., Trouvé A. (2008). La politique de développement rural : bilan et perspectives. Séminaire Confédération Paysanne-INRA «Face aux nouveaux enjeux : quelles politiques agricoles, pour quels systèmes de production ? », Criquetot L'Esneval, 8-9 avril.

Berdot J.-P., Léonard J. (2006). Globalisation et gouvernance territoriale : une introduction. Économies et Sociétés, vol. 41, n 3-4, p. 333-348.

Berriet-Solliec M., Chabé-Ferret S. (dir.) (2011). Analyser les politiques de développement rural. Pour un pilotage plus efficace de l'intervention régionale? Rapport final du programme de recherche REGIAB, PSDR 2009-2011, INRA-Régions Bourgogne et Auvergne.

Berriet-Solliec M., Delorme H., Laurent C., Mouriaux M.-F., Mundler P., Perraud D. (2006). Regulation of agriculture: the Regions as a new locus for working towards territorial coherence between agricultural policies? Canadian Journal of Regional Science, $\mathrm{n}^{\circ} 29$, p. 55-68.
Berriet-Solliec M., Daucé P., Daubard J.-P. (2001). Développement rural : quelle place pour l'agriculture dans les politiques communautaires? Déméter 2002, « Nouveaux enjeux pour l'agriculture », Paris, Armand Colin, p. 125-193.

Boltanski L., Chiapello E. (1999). Le nouvel esprit du capitalisme. Paris, Gallimard.

Callois J.-M. (2007). Les limites du territoire, une application de la notion de rayon de confiance au développement territorial. $R e$ vue d'économie régionale et urbaine, $\mathrm{n}^{\circ} 5$, p. 811-830.

Callois J.-M. (2009). Facteurs sociologiques de la dynamique de coopération intercommunale : vers une adaptation fine des politiques de développement territorial ? Numéro spécial de la Revue Auvergne sur le développement territorial.

Chavance B. (2007). L'économie institutionnelle. Paris, La Découverte, coll. « Repères $\gg$.

Colletis G., Gilly J.-P., Leroux I, Pecqueur B., Perat J., Rychen F., Zimmermann J.-B. (1999). Construction territoriale et dynamiques productives. Sciences de la Société, $\mathrm{n}^{\circ} 48$, p. 35-54.

Colletis G., Pecqueur B. (1993). Intégration des espaces et quasi-intégration des firmes : vers de nouvelles rencontres productives ? Revue d'économie régionale et urbaine, $\mathrm{n}^{\circ} 3$, p. 489-508. 
RECHERCHE

Cooke P., Morgan. K. (1998). The associational economy: firms, regions and innovation. Oxford, Oxford University Press.

Duran P., Thoenig J.-C. (1996). L'état et la gestion publique territoriale. Revue française de sciences politiques, vol. $46, \mathrm{n}^{\circ} 4$.

Dwyer J., Ward N., Lowe P., Baldock D. (2007). European Rural Development under the Common Agricultural Policy's "Second Pillar": Institutional Conservation and Innovation. Regional Studies, vol. $41 \mathrm{n}^{\circ} 7$, p. 873-887.

Fournier j. (2000). Le parc Livradois-Forez et les programmes Leader. Pour, $\mathrm{n}^{\circ} 167$.

Gaignette A., Nieddu M. (2000). L'agriculture française entre logiques sectorielles et territoriales (1960-1985). Cahiers d'économie et sociologie rurales, $\mathrm{n}^{\circ} 54$, p. 48-87.

Haskins (2003). Rural delivery Review: a report on delivery of government policies in rural England. DEFRA.

Jones M. (1998) Restructuring the local state: economic governance or social regulation? Political Geography, vol. 17, $\mathrm{n}^{\circ} 8$, p. 959-988.

Keating M. (1997). Les régions constituentelles un niveau de gouvernement en Europe ? In Le Galès P., Lequesne P. « Les paradoxes des régions en Europe », Paris, La Découverte, p. 19-35.

Knickel K., Jahn N. (2006). Promoting a sustainable development of rural areas: some relevant experiences with the "active regions" pilot programmes in Germany. In Remmers G. (dir.), "Moving Worldviews, Compas/ETC Foundation", Leusden.

Kroll J.-C. (2002). Nouvelles orientations de la politique agricole française : quelques questions à propos du CTE. Économie rurale, $n^{\circ} 268-269$, p. 32-44.

Krugman P. (1991). Increasing returns and economic geography. Journal of Political Economy, $\mathrm{n}^{\circ}$ 99, p. 483-499.

Laurent C., Du Tertre C. (dir.) (2008). Secteurs et territoires dans les régulations émergentes. Paris, L'Harmattan.

Lamarche T. (2008). Rupture dans la trajectoire historique du système éducatif : vers une logique sectorielle. In Laurent C., du Tertre C. « Secteurs et territoires dans les régulations émergentes », Paris, L'Harmattan.

Lovering J. (2001). The Coming Regional Crisis (and How To Avoid It). Regional Studies, vol. 35, $\mathrm{n}^{\circ} 4$, p. 349-354.

Maurel M.-C., Chevalier P., Pola P. (2010). L'expérimentation de l'approche LEADER en Hongrie, études de GAL en Baranya. In Maurel M.-C., Halamska M. (dir.), Le programme LEADER à l'épreuve du modèle européen, Publication du CEFRES et de l'IRWiR PAN de l'Académie des Sciences de Pologne, Prague, Varsovie, p. 71-93.

Pecqueur B. (2001). Gouvernance et régulation : un retour sur la nature du territoire. Géographie, Économie, Société, vol. 3, n² p. 229-245.

Rawls J. A. (19997) Théorie de la justice. Paris, Seuil.

Région Pays-de-la-Loire (2007). État des lieux des contrats de territoires. Rapport.

Rivet G. (2007). Le social et le politique face au territoire. In Bouquet B., Madelin B., Nivolle P. (dir.), Territoires et action sociale, Paris, L'Harmattan, p. 67-82.

Sapir A. (2003). An agenda for a growing Europe. Bruxelles, Commission européenne.

Sen A. (2000). Un nouveau modèle économique. Paris, Odile Jacob.

Shucksmith M. (2000). Endogenous development, social capital and social inclusion: perspectives from Leader in the UK. Regional studies, vol. $40, \mathrm{n}^{\circ} 2$.

Trouvé A. (2007). Le rôle des régions européennes dans la redéfinition des politiques agricoles. Doctorat en sciences économiques, Université de Bourgogne, UFR de sciences économiques, Dijon.

Trouvé A., Berriet-Solliec M., Déprès C. (2007). Charting and theorizing the territorialisation of agricultural policy. Journal of Rural Studies, $\mathrm{n}^{\circ} 23$, p. 443-452.

Webb D., Collis C. (2000). Regional development agencies and the 'new' regionalism in England. Regional Studies, vol. 34, n ${ }^{\circ} 9$, p. $857-873$. 